\title{
Claire Tardieu, Notions-clés pour la didactique de l'anglais
}

Paris : Presses Sorbonne Nouvelle

\section{Rebecca Starkey-Perret}

\section{CpenEdition}

\section{Journals}

Édition électronique

URL : http://journals.openedition.org/asp/4748

DOI : $10.4000 /$ asp. 4748

ISSN : 2108-6354

\section{Éditeur}

Groupe d'étude et de recherche en anglais de spécialité

Édition imprimée

Date de publication : 23 octobre 2015

Pagination : 139-142

ISSN : 1246-8185

\section{Référence électronique}

Rebecca Starkey-Perret, "Claire Tardieu, Notions-clés pour la didactique de l'anglais », ASp [En ligne], 68 | 2015, mis en ligne le 23 octobre 2015, consulté le 02 novembre 2020. URL : http:// journals.openedition.org/asp/4748; DOI : https://doi.org/10.4000/asp.4748

Ce document a été généré automatiquement le 2 novembre 2020

Tous droits réservés 


\section{Claire Tardieu, Notions-clés pour la didactique de l'anglais}

Paris : Presses Sorbonne Nouvelle

Rebecca Starkey-Perret

\section{RÉFÉRENCE}

Tardieu, Claire. 2014. Notions-clés pour la didactique de l'anglais. Paris : Presses Sorbonne Nouvelle. 236 pages. ISBN 978-2-87854-648-4. 
1 L'ouvrage de Claire Tardieu se donne pour objectif de permettre au lecteur de mieux maîtriser les termes parfois complexes de la didactique des langues, mais aussi et surtout, de mieux connaître les recherches qui les ont fait émerger.

2 Le livre, de 236 pages, comprend un préambule, une préface, une liste d'abréviations, une bibliographie, une table des figures et des tableaux ainsi que deux index, l'un de noms propres, l'autre de notions complémentaires. Il est organisé selon vingt-huit notions complexes, présentées par ordre alphabétique. Chaque notion est associée à plusieurs termes clés qui sont préalablement définis puis font l'objet d'une discussion théorique. Ainsi sont associés à chaque "notion " des termes qui sont liés ou antinomiques

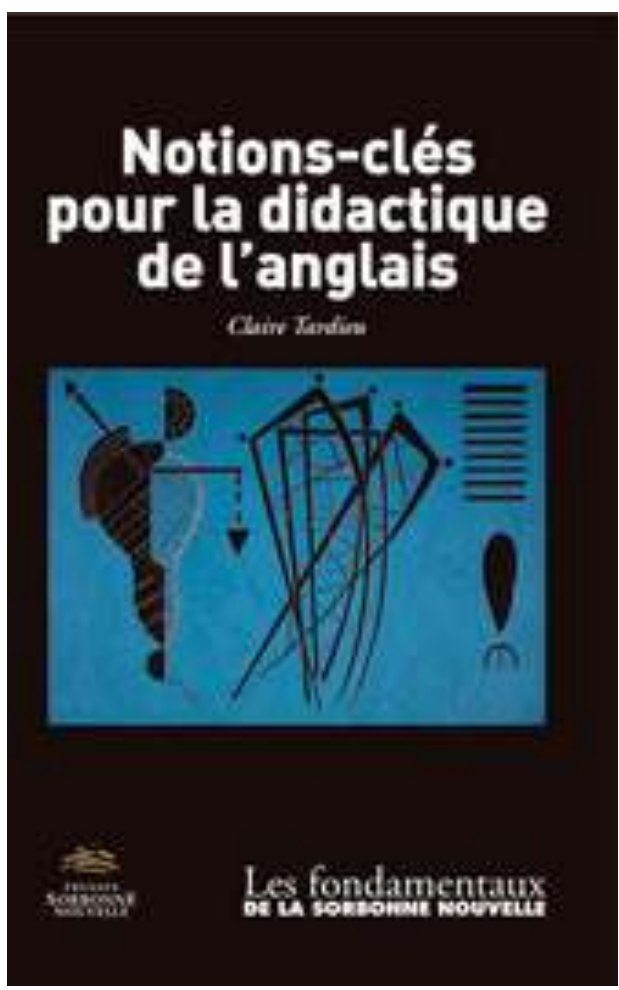
(Authentique-Didactisé-Pédagogique par exemple).

3 Une telle organisation des notions permet de relier des termes qui se trouveraient cloisonnés si l'ouvrage était présenté comme un dictionnaire de didactique des langues. En procédant de la sorte, l'auteure problématise chaque notion qui est ainsi montrée " sous toutes ses facettes" (p. 16). De cette manière, le livre cherche à aller au-delà de simples définitions pour aider le lecteur à construire sa réflexion et son recul épistémique sur la didactique des langues. Ce choix lui permet de réfléchir de manière informée, au lieu d'obtenir des réponses toutes faites, descendantes et peu satisfaisantes aux questions complexes du monde de l'enseignement/apprentissage des langues. Claire Tardieu met en pratique ce qu'elle formule ainsi en préambule :

La démarche proposée ici est donc moins définitoire qu'exploratoire, fondée non pas dans sur une réflexion qui segmente et divise que sur une réflexion qui rassemble et relie. (p. 13)

4 En même temps qu'il sert de guide utile pour conceptualiser les termes qui relèvent du domaine de la didactique des langues, cet ouvrage donne un aperçu panoramique des principales thématiques qui intéressent les enseignants et enseignants-chercheurs du domaine et les débats qui les entourent, telles que l'approche par les tâches, l'authenticité (des documents), le feedback correctif, la place de la grammaire, la mémoire, la précision et l'aisance, les représentations, le plurilinguisme ou encore les TIC.

5 Bien que le livre s'intitule Notions-clés pour la didactique de l'anglais, il peut, nous semble$\mathrm{t}$-il, être utilisé en didactique des langues dans son ensemble. La bibliographie comporte des références en anglais et en français, ce qui donne un aperçu international et actuel des notions abordées.

6 On regrettera qu'une part plus importante ne soit pas dévolue à l'anglais de spécialité et, par là, aux enseignants de l'enseignement supérieur qui interviennent dans les IUT, 
les facultés de sciences, les départements de psychologie, etc., en somme, le secteur LANSAD puisque nombre de notions abordées dans le livre sont susceptibles d'intéresser les chercheurs qui travaillent avec ce public d'apprenants. Les acronymes DNL, EMILE, CLIL, désignant l'enseignement intégré d'une matière et d'une langue dans le secondaire ou dans le supérieur, ne sont pas restreints à l'enseignement de l'anglais, mais renvoient aux langues. Il aurait été intéressant de trouver certains termes liés à l'anglais de spécialité, tels que EMI (English Medium Instruction) et le néologisme EMEMUS (English-Medium Education in Multilingual University Settings) qui, selon les chercheurs qui l'ont forgé, permettent de mieux se rendre compte de la réalité émergente de l'enseignement supérieur européen (Smit \& Dafouz 2012). Néanmoins, les termes EMILE, CLIL, LANSAD et LSP sont traités en distinguant la place de la langue par rapport à la matière. Les termes ASP/ESP (Anglais de Spécialité, English for Specific Purposes) sont également abordés dans le corps du texte, ce qui amène à une réflexion tout à fait pertinente pour le public des enseignants en LANSAD sur le rôle d'autres langues et qui prend appui sur «le glissement épistémologique d'une conception monolingue de l'enseignement des langues à une conception plurilingue » (p. 96).

7 La notion de «Précision-Aisance » (accuracy-fluency) est analysée rapidement (en deux pages, p. 96-97). Si l'auteure évoque bien le questionnement clé autour des termes "précision" et "aisance", qui constituent deux des trois "paramètres qui soustendent la compétence en L2 » selon Julie McAllister et Sophie Belan (2014 : 44), elle n'aborde pas en profondeur le troisième paramètre, celui de la notion de " complexité " (lexicale et syntactique), explorée notamment par Peter Skehan (2009). Selon Kate Wolfe-Quintero et al. (1998), la mesure de l'aisance, ou fluidité, dans la production des apprenants permet de rendre compte du degré d'automaticité du rappel des savoirs/ connaissances stockés en mémoire à long terme (DeKeyser 2001) tandis que les mesures de la précision et de la complexité concernent l'état de l'interlangue de l'apprenant (Selinker 1972). Les chercheurs qui s'intéressent à ces trois paramètres se posent la même question : comment viser à la fois l'aisance, la précision et la complexité ? Leurs travaux montrent qu'il est rare que les apprenants progressent simultanément dans ces trois domaines « car les ressources attentionnelles et la capacité de mémoire de travail chez les apprenants de L2 sont limitées " (McAllister \& Belan 2014 : 45). Dans le cadre de cet ouvrage, il n'est pas fait référence à ces recherches et l'accent est plutôt mis sur les définitions données dans le CECRL.

Dans la notion «Méthodes-Méthodologies », l'auteur se réfère principalement à la différence établie par Christian Puren (1988 : 17) entre méthodologie et méthode :

[...] selon lui, le terme de méthodologie s'applique à des formations historiques relativement différentes les unes des autres parce qu'elles se situent à un niveau supérieur où sont pris en compte des éléments sujets à des variations historiques déterminantes tels que les objectifs généraux [...] les contenus linguistiques et culturels [...] les théories de références [...]. (p.146)

C. Tardieu précise que la méthode découle de la méthodologie, mais reconnaît que le terme méthode est souvent utilisé « de manière générique pour distinguer à la fois les grands courants didactiques» (Puren 1988: 146), que Jack C. Richards et Theodore S. Rogers (1987) nomment "approche ", et «l'ensemble des procédés privilégiés par ces courants » (p. 146), désignés à travers les termes "méthode » ou encore, " technique " par ces mêmes auteurs. Ainsi, dans un tableau récapitulant les grands courants historiques de l'enseignement/apprentissage (p.147-149), les termes "méthode » et « approche » sont utilisés indifféremment tandis que d'autres chercheurs provenant 
d'une culture autre que la culture didactique française auraient tendance, en suivant la catégorisation proposée par J.C. Richards et T.S. Rogers (1987 : 37), à situer l'approche au niveau des théories linguistiques et psychologiques qui sous-tendent le choix des principes (méthodologie) et des pratiques (méthodes ou techniques) dans l'enseignement des L2. Si la catégorisation des termes n'est pas la même d'un chercheur à l'autre, on se rend compte qu'au niveau conceptuel, il y a en réalité consensus dans la hiérarchisation: la manière d'agir dans la situation d'apprentissage (techniques et matériels) découle de principes élaborés (consciemment ou non) par rapport aux représentations sur l'objet langue et sur la manière dont on l'acquiert.

Pour conclure, Notions-clés pour la didactique de l'anglais peut être considéré comme une mise à jour nécessaire de l'ouvrage de Danielle Bailly (1998). C'est cette chercheuse d'ailleurs qui en a rédigé la préface. Je partage son avis lorsqu'elle écrit que le premier mérite de l'ouvrage de Claire Tardieu est qu'il est actuel et que son deuxième réside dans le choix des notions à explorer car elles sont non seulement pertinentes dans la réflexion actuelle en didactique des langues, mais aussi souvent sujettes à des confusions « de par leur polysémie ou de par la difficulté de compréhension qu'ils sont susceptibles d'entraîner" (p. 16). À ces deux mérites j'ajouterais, en tentant de me positionner du point de vue d'un professeur de lycée ou de collège, que, même si l'ouvrage est étoffé et problématisé, chaque notion complexe est traitée de manière succincte, en trois à cinq pages seulement. Le livre est donc utile pour ceux qui manqueraient de temps pour faire un travail de recherche bibliographique approfondi ; il permet aussi à ceux qui souhaitent aller plus loin de repérer les lectures récentes les plus pertinentes pour explorer chaque notion. L'équilibre entre recherche et pratique (réflexive) de terrain, entre épistémologie et praxéologie, est ainsi maintenu tout au long de l'ouvrage.

11 Comme précisé dans le préambule, cet ouvrage de Claire Tardieu, Professeure à l'Université Sorbonne Nouvelle Paris 3, s'adresse aussi bien aux étudiants des masters MEEF et aux enseignants de langues du secondaire ou du supérieur, qu'aux doctorants et aux chercheurs dans le domaine de la didactique des langues.

\section{BIBLIOGRAPHIE}

BAILly, Danièle. 1998. Les Mots de la didactique des langues : le cas de l'anglais. Paris : Ophrys.

DEKEYSER, Robert M. 2001. « Automaticity and automatisation ». In Robinson, P. (dir.) Cognition and Second Language Instruction. Cambridge : Cambridge University Press, 125-151.

MCALLISTER, Julie \& Sophie BELAN. 2014. « L'anglais de spécialité en LEA à la croisée des domaines : étude de l'acquisition du lexique spécialisé ». ASp 66, 41-59.

PUREN, Christian. 1988. Histoire des méthodologies de l'enseignement des langues. Paris : Nathan, coll. CLE International. 
RICHARDS, Jack C. \& Theodore C. ROGERS. 1987. Approaches and Methods in Language Teaching. Cambridge : Cambridge University Press.

SELINKER, Larry. 1972. « Interlanguage ». IRAL 10/3, 209-231.

SKEHAN, Peter. 2009. « Modelling second language performance: Integrating complexity, accuracy, fluency and lexis ». Applied Linguistics, Special Issue: Complexity, Accuracy and Fluency (CAF) in Second Language Acquisition Research 30/4, 510-532.

SMIT, Ute \& Emma DAFOUZ (dir.). 2012. « Integrating Content and Language in Higher Education: Gaining Insights into English-Medium Instruction at European Universities ». AILA Review 25. Amsterdam : Benjamins.

WOLFE-QUINTERO, Kate, Shunji INAGAKI \& Hae-Young KIM. 1998. Second Language Development in Writing: Measures of Fluency, Accuracy and Complexity. Honolulu : University of Hawaii Press.

\section{AUTEURS}

\section{REBECCA STARKEY-PERRET}

Université de Nantes, Laboratoire CRINI, rebecca.starkey1@univ-nantes.fr 